\title{
A Comprehensive Analysis of Histopathologic Examination Results of Tonsillectomy Specimens
}

\author{
Serkan Kayabasi ${ }^{1}$, Omer Hizli ${ }^{2}$, Serkan Cayir ${ }^{3}$, Melike Ordu ${ }^{4}$ \\ 1. Otolaryngology, Faculty of Medicine, Aksaray University, Aksaray, TUR 2. Otolaryngology, Prof. Dr. A. Ilhan Ozdemir \\ Education and Research Hospital, Giresun University, Giresun, TUR 3. Otolaryngology, Aksaray Education and \\ Research Hospital, Aksaray University, Aksaray, TUR 4. Pathology, Faculty of Medicine, Aksaray University, Aksaray, \\ TUR
}

Corresponding author: Serkan Kayabasi, serkankayabasi@hotmail.com

\section{Abstract \\ Objective}

This study aims to review the histopathologic results of tonsillectomy specimens, determine the rates of the tonsillectomy indications, and investigate the characteristics of asymmetric hypertrophy.

\section{Materials and Methods}

Medical records of 484 patients who underwent tonsillectomy were reviewed retrospectively. Descriptive data of adult and pediatric patients were presented as percentage. Comparisons between asymmetric and symmetric hypertrophy groups were performed to determine the features of asymmetric hypertrophy.

\section{Results}

The mean age of 484 patients who underwent tonsillectomy was 13 years (range: 3-69 years). While 372 (76.85\%) patients were operated for infection, 100 (20.66\%) were operated for tonsillar hypertrophy, 1 (0.21\%) for a suspicion of malignancy, and 11 (2.27\%) for other various reasons. Asymmetric hypertrophy was seen in 25 (5.16\%) patients, whereas symmetric hypertrophy was seen in 75 (15.49\%) patients. Malignancy was detected in three ( $0.61 \%)$ adult patients with asymmetric hypertrophy. Tonsillar tuberculosis was observed in one foreign patient with asymmetric hypertrophy. The presence of malignancy was higher in the asymmetric hypertrophy group (three patients [12\%]) compared with the symmetric hypertrophy group (none) $\left(\mathrm{p}=0.002 ; \mathrm{X}^{2}=9.27\right)$. Median maximum specimen diameter was $3 \mathrm{~cm}$ (range: 1.15 $5.5 \mathrm{~cm}$ ) in the asymmetric hypertrophy group and $2.4 \mathrm{~cm}$ (range: $1.25-4.8 \mathrm{~cm}$ ) in the symmetric hypertrophy group $(\mathrm{p}=0.08)$. The Friedman grade was significantly $(\mathrm{p}<0.001)$, positively, and strongly $(\mathrm{r}=0.885)$ correlated with the maximum specimen diameter.

\section{Conclusion}

Received 01/09/2020

Routine histopathologic examination of the tonsillectomy specimens might not be necessary for all patients, but it is recommended for the patients with a real asymmetry.

Categories: Otolaryngology, Pathology

Keywords: tonsillectomy, histopathology, malignancy, asymmetry, hypertrophy

\section{Introduction}

Tonsillectomy is one of the most often performed operations in daily otolaryngology practice [1]. Tonsillectomy indications can be categorized as relative and definite. Recurrent tonsillitis, obstructive sleep apnea, peritonsillar abscess, halitosis, and suspicion of malignancy are the most common tonsillectomy indications. The most common indication in adults might be chronic recurrent tonsillitis; however, tonsillectomy has been gradually further performed as a part of the surgical treatment of obstructive sleep apnea in recent years [2]. There is no clear consensus on whether the histopathologic examination of clinically good-looking tonsil specimens is required [3]. Tonsillar malignant tumors might be clinically suspected of, but biopsy is required for certain histopathologic diagnoses. Therefore, tonsillectomy is usually performed for biopsy purposes in patients with a suspicion of malignancy. In the studies with large series, the risk of occult malignancy was reported to be quite lower in children [4]. The most important malignancy observed in children is usually tonsillar non-Hodgkin's lymphoma [5]. In adult patients, the incidence of malignancy after tonsillectomy varies from $2 \%$ to $10 \%$ [6]. The most common tonsillar malignancy is squamous cell carcinoma, which constitutes the majority of cases [7]. While squamous cell carcinoma occurs with an ulceration over the tonsil, basal cell carcinoma usually occurs with a normal mucosal and tonsillar asymmetry. Diagnosis of malignancy is very difficult in patients with normal tonsillar mucosa and without cervical adenopathy and/or additional physical examination findings [8]. 
In this study, we evaluated the histopathologic examination results of the patients who underwent tonsillectomy and determined the rates of the indications for tonsillectomy. The aim of the study was to determine the prevalence of various significant pathologies in patients with clinically asymmetric tonsils and to discuss the necessity of diagnostic tonsillectomy in patients with asymmetric tonsils.

\section{Materials And Methods \\ Study Design}

This retrospective study was conducted in line with the dictates of the World Medical Association Declaration of Helsinki and was approved by the local ethical committee (IRB Number: E-18-2420). The study included 484 patients who underwent tonsillectomy due to various indications (968 specimens) between April 2012 and January 2019. The ages, genders, tonsillectomy indications, histopathologic examination results, and clinical findings of the patients were noted by reviewing the medical records. According to the preoperative physical examination of all patients, the size of the tonsil was graded based on the grading system described by Friedman et al. [9]. According to this system, 0 (inside the tonsillar plica) was considered as atrophy, 1+ (at the same level as the tonsillar plica) was considered as normal, and 2+, 3+, and $4+$ (tonsils that close the oropharyngeal passage more than $25 \%, 50 \%$, and $75 \%$, respectively) were considered as hypertrophy. Those with a difference of grade over 1+ between the right and left sides were considered as asymmetric tonsillar hypertrophy (ATH). The surgical tonsillectomy method was cold dissection or thermal welding. Macroscopic and light microscopic histopathologic examination of the left and right tonsils was performed separately. The maximum diameter values of specimens were obtained from histopathologic examination result reports.

Tonsillectomy indications were categorized as chronic and/or recurrent infection, upper airway obstruction, suspicion of malignancy, and other reasons [1]. Patients with seven or more attacks of tonsillitis in the past year, those with five or more attacks of tonsillitis in the past two consecutive years, and those with three or more attacks of tonsillitis in the past three consecutive years were included in the first group as recurrent tonsillitis. The patients with snoring, open mouth sleeping, cessation of breathing during sleep in the noninfectious period, three or three attacks of tonsillitis in a year, and asymmetric or symmetric hypertrophy were included in the second group. The patients who were suspected of malignancy were included in the third group. Rare cases such as halitosis, tonsillolithiasis, tonsillar cyst, and tonsil bleeding were considered as the other reasons.

We constituted two groups by reference to the presence of asymmetric hypertrophy: ATH group and symmetric tonsillar hypertrophy (STH) group. We compared the presence of malignancy, actinomycosis, reactive lymphoid hyperplasia, and chronic inflammation between the groups. Additionally, we compared the mean ages, gender distribution, and median maximum specimen diameters of the groups. Also, to investigate whether preoperative Friedman grading was accurate, we performed a correlation test between the Friedman grade and maximum specimen diameter.

\section{Statistical Analysis}

We presented the results as median (min-max) and percentage (\%). We confirmed the abnormal distribution of data using the Kolmogorov-Smirnov normality test. To investigate the presence of malignancy, actinomycosis, reactive lymphoid hyperplasia, and chronic inflammation, we used the chi-square test. To compare the maximum specimen diameters of the groups, we used the Mann-Whitney U test. To investigate the correlation between the Friedman grade and maximum specimen diameter, we used Spearman's correlation test because Friedman grade data were ordinal. To compare the difference of maximum diameters of the right and left tonsil specimens (addressing asymmetry), we used the Mann-Whitney U test. We performed all statistical analyses using SPSS 16.0 software for Windows (SPSS Inc., Chicago, IL, USA). A p-value of less than 0.05 was considered statistically significant.

\section{Results}

The mean age of 484 patients was 13 years (range: 3-69 years), Of whom 282 (58.6\%) were males and 202 (41.7\%) were females. Of the patients, 411 (84.91\%) were under the age of 18 years (pediatric group), and 73 (15.08\%) were over the age of 18 years (adult group). The mean ages of children and adults were 7.1 years (range: 3-17 years) and 29.3 years (range: 18-69 years), respectively.

While 372 (76.85\%) patients underwent tonsillectomy for chronic/ recurrent infection, 100 (20.66\%) were operated for tonsillar hypertrophy, $1(0.21 \%)$ for a suspicion of malignancy, and $11(2.27 \%)$ for other reasons. The distribution of tonsillectomy indications in the pediatric and adult patients are shown in Table 1 . 


\section{Cureus}

\begin{tabular}{|l|lll|l|}
\hline Indications & \multicolumn{2}{l|}{ Pediatric patients } & \multicolumn{2}{l|}{ Adult patients } \\
\cline { 2 - 4 } & $\mathrm{n}$ & $\%$ & $\mathrm{n}$ & $\%$ \\
\hline Infections & 312 & $75.91 \%$ & 60 & $82.2 \%$ \\
Obstruction & 95 & $23.12 \%$ & 5 & $6.85 \%$ \\
Suspicion of malignancy & 0 & $0 \%$ & 1 & $1.37 \%$ \\
Other reasons & 4 & $0.97 \%$ & 7 & $9.58 \%$ \\
Total & 411 & 100 & 73 & 100 \\
\hline
\end{tabular}

TABLE 1: The distribution of tonsillectomy indications in the pediatric and adult patients

Among the patients operated for tonsillar hypertrophy, ATH was detected in 25 (5.16\%) patients, whereas STH was detected in 75 (15.49\%) patients. Adult tonsillar squamous cell carcinoma was detected in one $(0.21 \%)$ patient operated for a suspicion of malignancy. With respect to the other indications, two patients $(0.41 \%)$ had tonsil bleeding, three $(0.61 \%)$ had tonsillolithiasis with halitosis, three $(0.61 \%)$ had tonsillar papilloma, and three (0.61\%) had tonsil cyst.

Of 484 (968 specimens) patients who underwent tonsillectomy, 219 (45.24\%) had reactive lymphoid hyperplasia, 137 (28.30\%) had reactive lymphoid hyperplasia with chronic inflammation, and 97 (20.04\%) had chronic inflammation in their histopathologic examination result reports. Malignancy was detected only in the specimens of three (0.30\%) patients. All these patients were in the adult patient group. Malignancy was not evident in the pediatric patient group. Two patients with malignancy were diagnosed as having diffuse large B-cell non-Hodgkin lymphoma (Figure 1).

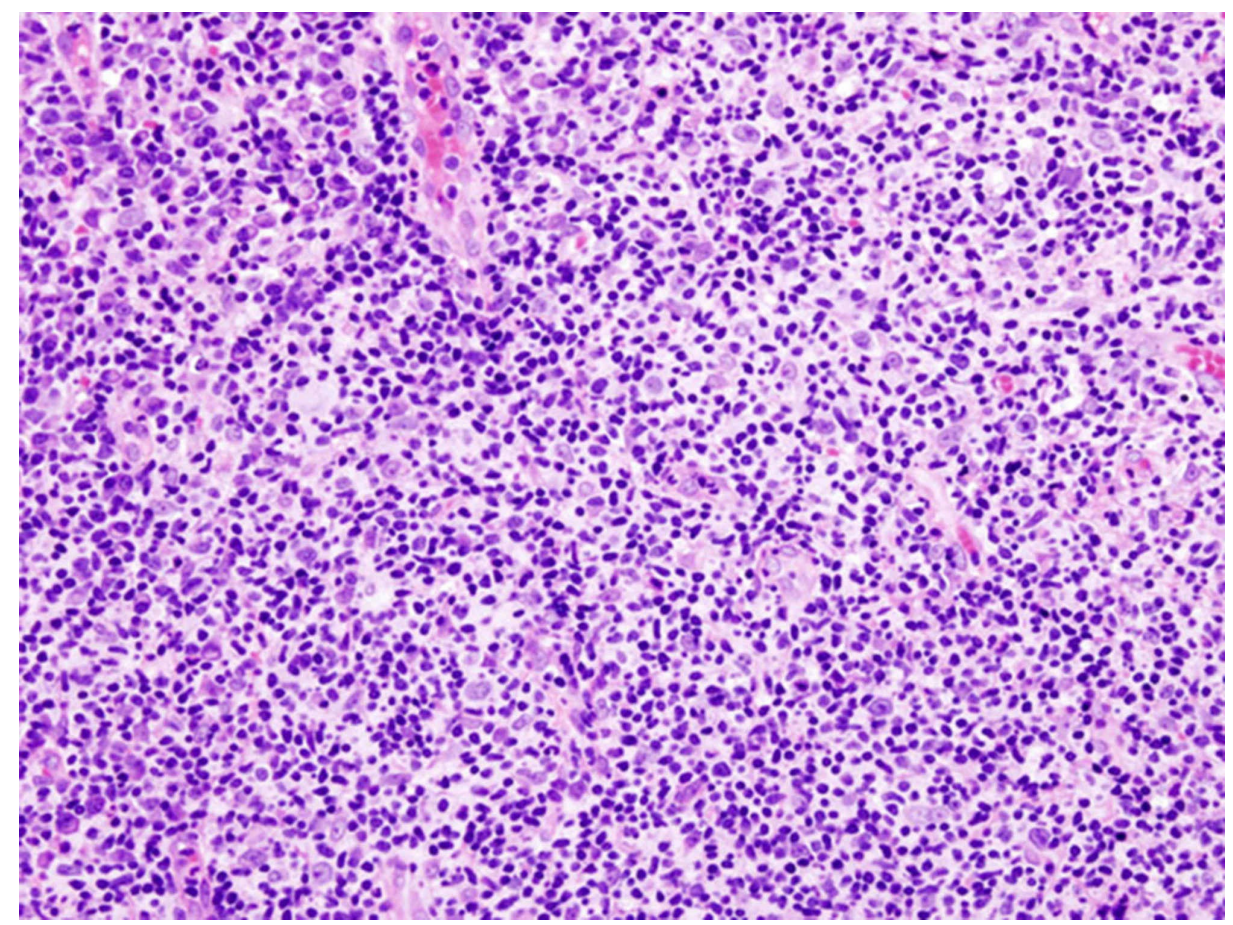

FIGURE 1: Light photomicrograph of histopathologic section from a patient with diffuse large B-cell non-Hodgkin lymphoma (hematoxylin and eosin stain, 100x magnification)

While unilateral tonsillar hypertrophy (asymmetric hypertrophy) was detected in these patients with lymphoma, normal tonsillar mucosa without any ulceration was seen in physical examination. The first patient was a 54-year-old woman with recurrent attacks of tonsillitis and left tonsillar hypertrophy. The other patient was a 64-year-old woman with right tonsillar hypertrophy. Both patients underwent 


\section{Cureus}

tonsillectomy for recurrent tonsillitis, not for any suspicion of malignancy. Thus, two $(0.41 \%)$ patients in our study group had an occult malignancy (without a preoperative suspicion). Additionally, occult malignancy was not seen in pediatric patients, and the incidence of occult malignancy was $2.7 \%$ in adult patients. In the physical examination of both patients, preoperative palpable lymphadenopathy findings were remarkable. In addition to the routine histopathologic examination, immunohistochemical evaluation was performed in both patients. The immunohistochemical staining revealed positive keratin, negative $C D 20$, and negative CD3 staining features in both patients. Out of 484 patients, only one (0.21\%) patient underwent tonsillectomy for a suspicion of malignancy. In the physical examination of this patient, ulceration and ATH were seen. Histopathologic examination result of this 69 -year-old male patient was reported as squamous cell carcinoma (Figure 2).

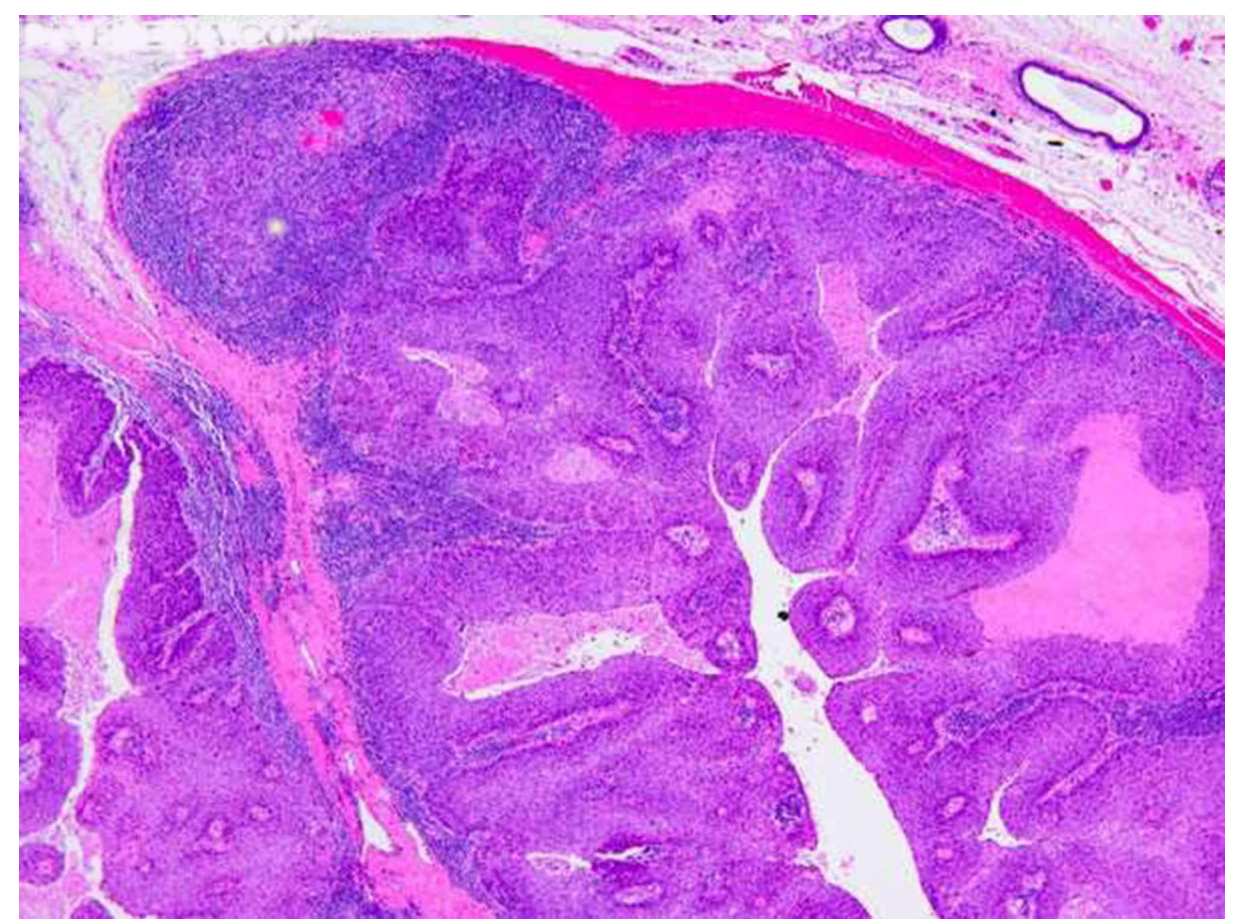

FIGURE 2: Light photomicrograph of histopathologic section from the patient with squamous cell carcinoma (hematoxylin and eosin stain, 10x magnification)

According to the analysis of histopathologic examination results, 22 patients had actinomycosis (14 pediatric patients and 8 adult patients) (Figure 3), 3 had squamous papilloma (2 pediatric patients and 1 adult patient), and 3 had epidermal inclusion cyst ( 2 pediatric patients and 1 adult patient). One adult patient with ATH had tonsillar tuberculosis (Table 2). 


\section{Cureus}

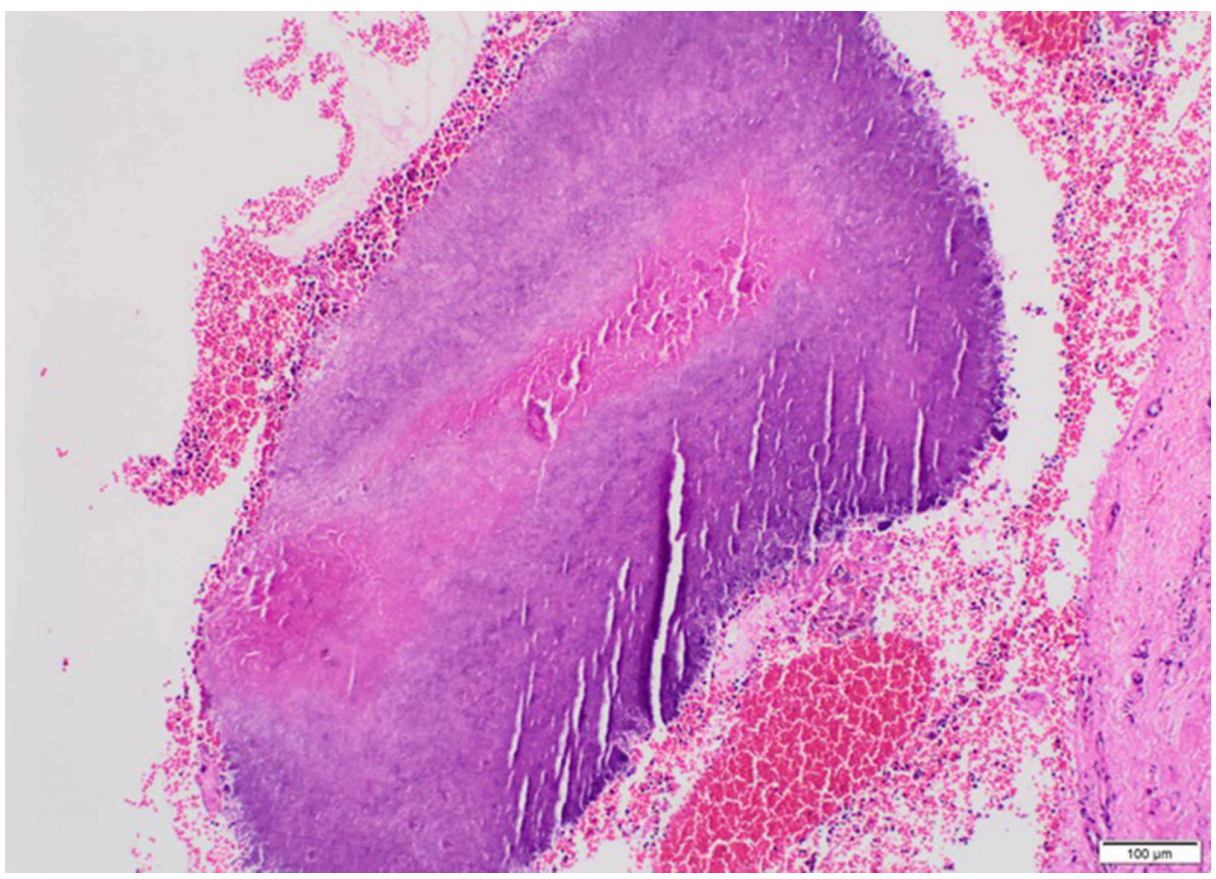

\section{FIGURE 3: Light photomicrograph of histopathologic section from the patient with tonsillar actinomycosis (hematoxylin and eosin stain, $\mathbf{x 1 0}$ magnification)}

Histopathologic diagnosis

Chronic inflammation

Reactive lymphoid hyperplasia

Chronic inflammation + reactive lymphoid hyperplasia

Malignancies

Actinomycosis

Tuberculosis

Squamous papilloma

Epidermal inclusion cyst

Total

\begin{tabular}{llllll}
\hline Pediatric patients & \multicolumn{2}{l}{ Adult patients } & \multicolumn{2}{l}{ Total } \\
$\mathrm{n}$ & $\%$ & $\mathrm{n}$ & $\%$ & $\mathrm{n}$ & $\%$ \\
70 & 17.03 & 27 & 36.98 & 97 & 20.04 \\
205 & 49.87 & 14 & 19.17 & 219 & 45.24 \\
118 & 28.71 & 19 & 26.02 & 137 & 28.30 \\
- & - & 3 & 2.19 & 3 & 0.61 \\
14 & 3.40 & 8 & 10.95 & 22 & 4.54 \\
- & - & 1 & 1.36 & 1 & 0.20 \\
2 & 0.48 & 1 & 1.36 & 3 & 0.61 \\
2 & 0.48 & 1 & 1.36 & 3 & 0.61 \\
411 & 84.91 & 73 & 15.08 & 484 & 100 \\
& & & & &
\end{tabular}

TABLE 2: Histopathologic examination results of our study population

Out of 484 patients who underwent tonsillectomy, 100 (20.6\%) had tonsillar hypertrophy. The ATH group consisted of 25 patients (13 males and 12 females; mean age: 16 years [range: 3-69 years]), and the STH group consisted of 75 patients ( 41 males and 34 females; mean age: 11 years [range: $3-44$ years]). The groups were age and gender-matched ( $\mathrm{p}=0.82$ and $\mathrm{p}=0.81$, respectively). While no ( $0 \%)$ malignancy was reported in histopathologic examination results of the patients from the STH group, three $(12 \%)$ malignancies were reported in the ATH group ( $\mathrm{X} 2=9.27 ; \mathrm{p}=0.02)$. One (4\%) patient in the ATH group and six $(8 \%)$ patients in the STH group had actinomycosis, thus not significantly different $(\mathrm{X} 2=0.46 ; \mathrm{p}=0.49)$. Reactive lymphoid hyperplasia was present in 17 (68\%) patients in the ATH group and 61 (81.3\%) patients in the STH group, also not significantly different $(\mathrm{X} 2=1.94 ; \mathrm{p}=0.16)$. Chronic inflammation was present in $8(32 \%)$ patients in the ATH group and 34 (45.3\%) patients in the STH group, again not significantly different (X2=1.36; $p=0.24$ ) (Table 3). Thus, ATH was associated with tonsillar malignancies but not with actinomycosis, reactive lymphoid hyperplasia, and chronic inflammation. 


\section{Cureus}

\begin{tabular}{|c|c|c|c|c|}
\hline Clinicopathological findings & $\begin{array}{l}\text { ATH group, } \\
n=25\end{array}$ & $\begin{array}{l}\text { STH group, } \\
n=75\end{array}$ & $\begin{array}{l}\text { p- } \\
\text { Value }\end{array}$ & $\mathrm{X}^{2}$ value \\
\hline Age, years & $16(3-69)$ & $11(3-44)$ & 0.82 & \\
\hline Gender (male/female) & $13 / 12$ & $41 / 34$ & 0.81 & \\
\hline Median maximum specimen diameter $(\mathrm{cm})$ & $3(1.15-5.5)$ & $2.4(1.25-4.8)$ & 0.008 & \\
\hline $\begin{array}{l}\text { Median difference of maximum specimen diameter between the right and left } \\
\text { tonsils }(\mathrm{cm})\end{array}$ & $1.20(0.5-3.4)$ & $0.15(0-1.45)$ & $<0.001$ & \\
\hline Malignancy & $3(12 \%)$ & $0(0 \%)$ & 0.02 & 9.27 \\
\hline Chronic inflammation & $8(32 \%)$ & $34(45.3 \%)$ & 0.24 & 1.36 \\
\hline Reactive lymphoid hyperplasia & $17(68 \%)$ & $61(81.3 \%)$ & 0.16 & 1.94 \\
\hline Actinomycosis & $1(4 \%)$ & $6(8 \%)$ & 0.49 & 0.46 \\
\hline
\end{tabular}

\section{TABLE 3: Comparison of clinicopathological findings of the groups}

ATH, asymmetric tonsillar hypertrophy; STH, symmetric tonsillar hypertrophy

The Friedman grade was significantly ( $\mathrm{p}<0.001)$, positively, and strongly $(\mathrm{r}=0.885)$ correlated with the maximum specimen diameter of tonsils. The median maximum specimen diameter was $3 \mathrm{~cm}$ (range: 1.15 $5.5 \mathrm{~cm}$ ) in the ATH group and $2.4 \mathrm{~cm}$ (range: $1.25-4.8 \mathrm{~cm}$ ) in the STH group. Patients with ATH had significantly greater maximum specimen diameter $(\mathrm{p}=0.008)$. The median difference of maximum diameters between the right and left tonsils was $1.20 \mathrm{~cm}$ (range: 0.5-3.4 cm) in the ATH group and $0.15 \mathrm{~cm}$ (range: 0$1.45 \mathrm{~cm})$ in the STH group, thus significantly greater in the ATH group $(\mathrm{p}<0.001)$ as expected.

\section{Discussion}

Tonsillectomy (with or without adenoidectomy) is one of the most common operations performed in children [10]. Tonsillectomy has been previously performed to avoid the complications of group A betahemolytic streptococcal infection; however, it is now performed mainly for chronic and/or recurrent infection and obstructive reasons [11]. In this study, while 372 (76.85\%) patients underwent tonsillectomy due to the diagnosis of chronic and/or recurrent infection, 100 (20.66\%) underwent tonsillectomy for upper airway obstruction due to tonsillar hypertrophy.

Obstructive causes were reported to increase in recent years among tonsillectomy indications, particularly in pediatric patients. According to the report by Parker and Walner, obstruction was the primary tonsillectomy indication (91.8\%) in the 0-3 age group, of which only $7.5 \%$ underwent tonsillectomy for chronic recurrent infection. [12]. In contrast to the prior literature, $75.91 \%$ of pediatric patients underwent tonsillectomy due to the diagnosis of chronic and/or recurrent infection, whereas $23.12 \%$ underwent tonsillectomy due to the diagnosis of upper airway obstruction in our study. Similarly, the most common tonsillectomy indication was infectious causes in our adult patient group (60 [82.2\%] patients).

According to the prior literature, the most common tonsillar malignancy was squamous cell carcinoma in adults. Lymphomas were more frequently seen in children. The incidence of occult malignancy was reported as $0-1 \%$ in the literature [13]. In our study, malignancy was observed in one $(0.21 \%)$ patient who underwent tonsillectomy due to a suspicion of malignancy. This patient was a 69-year-old man with ATH, and his histopathologic examination result was reported as squamous cell carcinoma. The incidence of tonsillar malignancy was higher in adults compared with children, which might be due to smoking, alcohol consumption, and other environmental factors. In our study group, occult malignancy was seen in two (0.41\%) patients, consistent with the prior literature [13].

In our study, an epidermal inclusion cyst was detected in one adult patient and squamous papilloma was detected in another adult patient. Actinomycosis with chronic inflammation was reported in eight adult patients. Tonsillar tuberculosis was detected in a foreign adult with ATH. Tonsillar tuberculosis is an extrapulmonary type of tuberculosis that can frequently mimic tonsillar malignancy and is rarely observed $(10-15 \%)$ [14]. Its secondary form is more common than its primary form. Contact with the infected sputum or saliva is the main source of the disease. Clinical and recurrent tonsillitis is the main clinical presentation with large asymmetric tonsils and sore throat. Since it is very difficult to distinguish it from malignancy clinically, the histopathologic examination of the tissue is required for certain diagnoses. Tonsillar tuberculosis is also not a common entity in Turkey; thus, our results suggest that migrations and exchanges may affect the infectious disease profile in a country. 
Malignancy was not observed in our pediatric patient group. In the previous reports, malignancy rates ranged from $0 \%$ to $0.17 \%$ in the pediatric population [15]. Although tonsillar malignancy rates in pediatric patients are lower, a detailed histopathologic examination should be performed in patients with preoperative risk factors such as necrotic tonsil, lymphadenopathy on the neck, weight loss, night sweating, and tonsillar asymmetry. In our study, histopathologic examination was reported as epidermal inclusion cyst in two pediatric patients and as squamous papilloma in two pediatric patients. These patients had preoperative well-defined benign lesions, and histopathologic findings were consistent with clinical findings. The presence of actinomycosis in tonsil specimens was reported as $1.3-57 \%$ [16]. In our study, actinomycosis with chronic inflammation was observed in 14 pediatric patients. Many previous studies claimed that actinomycosis might play a role in the etiology of chronic tonsillitis and adenoid vegetation, although it was not definitely demonstrated [17].

Although histopathologic examination is usually performed after tonsillectomy in general practice, the necessity of histopathologic examination is a matter of debate. In addition, it is still unclear that in which cases histopathologic examination is required. Felippe et al. reviewed 2013 adult and pediatric patients who underwent adeno-tonsillectomy and reported malignancy in $4(0.19 \%)$ patients [18]. In the report by Younis et al., malignancy was not detected in 2099 pediatric patients and was detected in 40 (11.79\%) of 339 adult patients. The most common malignancy type was squamous cell carcinoma in this report [19]. In addition, they suggested that gross examination of specimens was sufficient and they required histopathologic examination in suspected cases [19]. In our study, malignancy was detected in two patients who underwent tonsillectomy for obstruction. These patients were in the adult group and diagnosed as having diffuse large B-cell non-Hodgkin lymphoma. Tonsillar asymmetry and lymphadenopathy on the neck were present in both patients, and normal mucosa without ulceration was seen in their physical examinations. These two patients with lymphoma could be considered as occult malignancy due to the absence of preoperative suspicion of malignancy. Based on our results, we can suggest that histopathologic examination is needed for the specimens of adult patients with ATH and any additional physical examination finding, such as lymphadenopathy of the neck, due to occult malignancy risk.

ATH is not a rare condition. Even though ATH is usually benign, tonsillar tumors should be taken into consideration at differential diagnosis. In our study, no malignancy was evident in patients with STH. Chronic inflammation, reactive lymphoid hyperplasia, and actinomycosis rates were higher in the STH group compared with the ATH group but did not significantly differ. This difference might have been significant if the ATH group consisted of a larger number of patients. Therefore, we found that ATH was associated with tonsillar malignancies in this study, but the evidence of association of STH with actinomycosis, reactive lymphoid hyperplasia, and chronic inflammation was not clear. Spinou et al. reported the malignancy rate of the patients with ATH as high as $23.4 \%$, as 23 malignancies were present in 98 adults with ATH in their study. Accordingly, they found a clinical suspicion of malignancy in each of these cases, such as ulceration, male gender, lymphadenopathy, age over 45 years, smoking, and weight loss [20].

In our study, we found a significant and strong correlation between the preoperative grade and maximum specimen diameter of excised tonsils. However, Cable et al reported that clinically assessed size was not correlated with the volume pathologically measured after tonsillectomy in children [21]. Berkowitz and Mahadevan found that only $52 \%$ of histopathologic examination results revealed a tonsillar size asymmetry in patients considered to have a preoperative large ATH [22]. According to Spinou et al., 17\% of the patients with clinically asymmetric tonsils had a smaller tonsil size and that the sizes of the right and left tonsils were equal in $40 \%$ of the patients [23]. Cinar did not find a difference in the tonsil size of $39.62 \%$ of the asymmetric group and concluded that apparent asymmetry might be due to the difference in the tonsillar fossa depth [8]. In contrast to these studies, Syms et al. reported that clinically assessed size was consistent with pathologically measured volume in $60.5 \%$ of the patients with ATH [24]. In our study, patients with ATH had significantly greater maximum specimen diameter compared with those with STH. Furthermore, the Friedman grade was significantly, positively, and strongly correlated with the maximum specimen diameter of the tonsils. This result shows that we performed an accurate preoperative Friedman grade evaluation or that the Friedman grading system [9] might be considered as an accurate grading system.

\section{Conclusions}

In conclusion, the routine histopathologic examination of the tonsillectomy specimens might not be necessary for all patients, but it is recommended for patients with a real asymmetry. Physicians should bear in mind the possibility of asymmetry due to variability in the tonsillar fossa. Cooperation of otolaryngology and pathology physicians for routine histopathologic examination after tonsillectomy would contribute to an early diagnosis of rare tonsillar malignancies in patients with a real asymmetry.

\section{Additional Information \\ Disclosures}

Human subjects: Consent was obtained by all participants in this study. Ankara Numune Education and Research Hospital issued approval E-18-2420. Animal subjects: All authors have confirmed that this study did not involve animal subjects or tissue. Conflicts of interest: In compliance with the ICMJE uniform 
disclosure form, all authors declare the following: Payment/services info: All authors have declared that no financial support was received from any organization for the submitted work. Financial relationships: All authors have declared that they have no financial relationships at present or within the previous three years with any organizations that might have an interest in the submitted work. Other relationships: All authors have declared that there are no other relationships or activities that could appear to have influenced the submitted work.

\section{References}

1. Baugh RF, Archer SM, Mitchell RB, et al.: Clinical practice guideline: tonsillectomy in children. Otolaryngol Head Neck Surg. 2011, 144:1-30. 10.1177/0194599810389949

2. Erickson BK, Larson DR, St Sauver JL, Meverden RA, Orvidas LJ: Changes in incidence and indications of tonsillectomy and adenotonsillectomy, 1970-2005. Otolaryngol Head Neck Surg. 2009, 140:894-901. 10.1016/j.otohns.2009.01.044

3. Bizzell JG, Richter GT, Bower CM, Woods GL, Nolder AR: Routine pathologic examination of tonsillectomy specimens: A 10-year experience at a tertiary care children's hospital. Int J Pediatr Otorhinolaryngol. 2017, 102:86-89. 10.1016/i.ijporl.2017.09.012

4. Booth CL, Wang J: Occult hematologic malignancy in routine tonsillectomy specimens: a single institutional experience and review of the literature. Am J Clin Pathol. 2013, 140:807-812. 10.1309/AJCPCM7LN4QEZPOQ

5. Paulsen J, Lennert K: Low-grade B-cell lymphoma of mucosa-associated lymphoid tissue type in Waldeyer's ring. Histopathology. 1994, 24:1-11. 10.1111/j.1365-2559.1994.tb01265.x

6. Papouliakos S, Karkos PD, Korres G, Karatzias G, Sastry A, Riga M: Comparison of clinical and histopathological evaluation of tonsils in pediatric and adult patients. Eur Arch Otorhinolaryngol. 2009, 266:1309-1313. 10.1007/s00405-008-0869-4

7. Strong EB, Rubinstein B, Senders CW: Pathologic analysis of routine tonsillectomy and adenoidectomy specimens. Otolaryngol Head Neck Surg. 2001, 125:473-477. 10.1067/mhn.2001.119862

8. Cinar F: Significance of asymptomatic tonsil asymmetry. Otolaryngol Head Neck Surg. 2004, 131:101-103. 10.1016/j.otohns.2004.02.004

9. Friedman M, Tanyeri H, La Rosa M, et al.: Clinical predictors of obstructive sleep apnea. Laryngoscope. 1999, 109:1901-1907. 10.1097/00005537-199912000-00002

10. Faramarzi A, Kadivar MR, Heydari ST, Tavasoli M: Assessment of the consensus about tonsillectomy and/or adenoidectomy among pediatricians and otolaryngologists. Int J Pediatr Otorhinolaryngol. 2010, 74:133136. 10.1016/j.ijporl.2009.10.024

11. Hoddeson EK, Gourin CG: Adult tonsillectomy: current indications and outcomes. Otolaryngol Head Neck Surg. 2009, 140:19-22. 10.1016/j.otohns.2008.09.023

12. Parker NP, Walner DL: Trends in the indications for pediatric tonsillectomy or adenotonsillectomy . Int J Pediatr Otorhinolaryngol. 2011, 75:282-285. 10.1016/j.ijporl.2010.11.019

13. Williams MD, Brown HM: The adequacy of gross pathological examination of routine tonsils and adenoids in patients 21 years old and younger. Hum Pathol. 2003, 34:1053-1057. 10.1053/S0046-8177(03)00408-8

14. Thiagarajan B, Ulaganathan V, Ramamoorthy G: Secondary Tuberculosis of Tonsil case report and literature review. Online J Otolaryngol. 2012, 52:60.

15. Kepekci AH, Balikci HH: Is routine histopathologic examination necessary following tonsillectomy and/or adenoidectomy procedures in pediatric patients?. J Craniofac Surg. 2017, 28:91-93. 10.1097/scs.0000000000003278

16. Toh ST, Yuen HW, Goh YH: Actinomycetes colonization of tonsils: a comparative study between patients with and without recurrent tonsillitis. J Laryngol Otol. 2007, 121:775-778. 10.1017/s0022215106003185

17. Aydin A, Erkilic S, Bayazit YA, Kocer NE, Ozer E, Kanlikama M: Relation between actinomycosis and histopathological and clinical features of the palatine tonsils: a comparative study between adult and pediatric patients. Rev Laryngol Otol Rhinol (Bord). 2005, 126:95-98.

18. Felippe F, Gomes GA, de Souza BP, Cardoso GA, Tomita S: Evaluation of the utility of histopathologic exam as a routine in tonsillectomies. Braz J Otorhinolaryngol. 2006, 72:252-255. 10.1016/s1808-8694(15)30064-1

19. Younis RT, Hesse SV, Anand VK: Evaluation of the utility and cost-effectiveness of obtaining histopathologic diagnosis on all routine tonsillectomy specimens. Laryngoscope. 2001, 111:2166-2169. 10.1097/00005537-200112000-00017

20. Spinou C, Kubba H, Konstantinidis I, Johnston A: Role of tonsillectomy in histology for adults with unilateral tonsillar enlargement. Br J Oral Maxillofac Surg. 2005, 43:144-147. 10.1016/j.bjoms.2004.01.009

21. Cable HR, Batch AG, Stevens DJ: The relevance of physical signs in recurrent tonsillitis in children (a prospective study). J Laryngol Otol. 1986, 100:1047-1051. 10.1017/S0022215100100544

22. Berkowitz RG, Mahadevan M: Unilateral tonsillar enlargement and tonsillar lymphoma in children . Ann Otol Rhinol Laryngol. 1999, 108:876-879. 10.1177/000348949910800910

23. Spinou E, Kubba H, Konstantinidis I, Johnston A: Tonsillectomy for biopsy in children with unilateral tonsillar enlargement. Int J Pediatr Otorhinolaryngol. 2002, 63:15-17. 10.1016/s0165-5876(01)00633-4

24. Syms MJ, Birkmire-Peters DP, Holtel MR: Incidence of carcinoma in incidental tonsil asymmetry . Laryngoscope. 2000, 110:1807-1810. 10.1097/00005537-200011000-00006 